\title{
Application of 4D CAD System for Infrastructure Projects with Construction Schedules and Distance Coordinates
}

\author{
Hyeon Seung-Kimª, Sang Mi-Park ${ }^{\mathrm{b}}$, Jae Hee-Lee ${ }^{\mathrm{c}}$, and Leen Seok-Kang* \\ ${ }^{a}$ Dept. of Civil Engineering, Gyeongsang National University, Jinju 52828, Korea (E-mail: wjdchs2003@gmail.com) \\ ${ }^{b}$ Dept. of Civil Engineering, Gyeongsang National University, Jinju 52828, Korea (E-mail: gogf8585@naver.com) \\ ${ }^{c}$ Dept. of Civil Engineering, Gyeongsang National University, Jinju 52828, Korea (E-mail: akfn1614@gmail.com) \\ *Professor, Dept. of Civil Engineering, Gyeongsang National University, ERI, Jinju 52828, Korea (Corresponding Author, E-mail: \\ Lskang@gnu.ac.kr)
}

\begin{abstract}
The typical constraints in expressing the current construction status of railway and road construction projects in a 4D CAD system are the complexity of creating 3D objects for many earthworks and non-repetitive processes. Also, since most of the work is done in a linear pattern in a horizontal work space of several tens of $\mathrm{km}$, there are some parts that are difficult to express by the commercialized 4D CAD system oriented on the building project. In the activity management of the railway construction, most activities such as earthwork, bridge, tunnel, and catenary line except railway station are managed by the distance of linear axis. In other words, a methodology is required in the 4D CAD system to grasp the distance coordinates of the activity in addition to the construction schedule even when 4D objects are implemented. In this paper, the authors propose an automatic transformation method to linear schedule chart of Gantt chart expressed in a 4D CAD system, and propose a methodology for specialized functions of 4D CAD system which converted linear schedule chart and 4D object are interlocked.
\end{abstract}

Keywords:infrastructure project; linear schedule chart; Gantt chart; 4D CAD ;

\section{Introduction}

Building construction project consists of repetitive works in a narrow space with a vertical work pattern. On the other hand, the civil engineering project is working horizontally, and work is carried out with non-repetitive works in a wide working area. This feature is the reason why the application of BIM in civil engineering project is relatively small compared to the building project. In addition, since the civil engineer project includes many earthwork tasks, it is more difficult to represent the work objects in a three dimension. Although it may be different depending on the type of construction project, the importance of the design stage is larger than the construction stage in the building construction project, and the civil engineer project has a greater importance in the construction phase. For this reason, 3D modeling and interference management at the design stage are important functions for the application of BIM to the building project, and schedule management at the construction stage can be used as an important function for the BIM application of the civil engineering project. In other words, BIM application of civil engineering project can be more easily utilized in the construction management function by 4D and nD CAD.

4D CAD systems used for construction schedule management integrate the construction schedule and 3D design plan to sequentially visualize the completed form of the facility and provide real-time visualized site information to the participants of the construction project. Using a 4D CAD system, the completed form of a facility can be sequentially displayed according to the schedule of planned activities. Through this, the construction work can be reviewed in advance before the construction phase in virtual reality, which can be used to carry out schedule error reviews in the planning stage and constructability and interference evaluation for the facility in advance. Thus, 
constructability for each actual site can be improved through simulations using 3D objects according to the schedule. $4 \mathrm{D}$ systems can be utilized as a tool to facilitate communication between construction participating parties for solution establishment and prevention of constructability reduction between parallel tasks.

Various studies on BIM have been conducted. The main contents of this study are composed of 4D CAD related matters, so the existing researches are also examined based on 4D CAD operating system. Leon (2016) proposed a 4D CAD based method to support coordination of subsurface utility works. Christopher (2018) suggested an interior activity state recognition framework with BIM-registered image sequences. Kang (2013) presented a methodology and system development methodology for 4D CAD operating system specialized in civil engineering project. Moon (2015) suggests a systematic methodology and computer system for an optimal construction schedule simulation that minimize overlapping activities using 4D CAD system. Chin (2008) developed a progress management system that links RFID technology and 4D CAD to steel structures for efficient logistics support and construction management. Ashwin (2010) proved the usability of 4D CAD by quantitatively and statistically analyzing 4D CAD cases applied to practical work in order to improve the practical use of 4D CAD. Timo (2008) suggested a way to analyze applications of 3D and 4D models through past cases and apply them effectively in practice. Russell et al. (2009) developed a 4D CAD system that can efficiently perform construction management tasks considering linear scheduling of high-rise buildings.

As the utilization of 4D CAD system is more active in the building project, many related researched are also applied to practical application such as interference management of building works. As the application of BIM technology is expanded, applications of AR technology are also being announced. In this case, the researches on the construction of $4 \mathrm{D}$ CAD function, which is composed of linear activity processes in the horizontal working area, are not enough. This study attempts to develop a function suitable for the simulation of a construction schedule consisting of linear space such as road and railway projects.

\section{Necessity of function improvement for existing 4D CAD system}

Various commercially available 4D CAD systems currently used in practice are all centered on the schedule simulation functions in a vertical work space. In other words, since it is used mainly as a function of time-based schedule simulation function, it lacks practical progress management function and lacks other decision-making functions. The following functions for $4 \mathrm{D} \mathrm{CAD}$ system are necessary to obtain practical use applicability in the construction phase of building and civil engineering construction projects.

- Intuitive progress management system: Provision of simulation information is necessary to intuitively determine the actual progress in comparison to the established schedule by comparing real video data such as that from CCTV installed at the construction site and smart phones with 4D objects.

- AR object integration function: Activities simulated with 4D objects are images expressed using VR (virtual reality) type computer graphics, and as such there is a discrepancy between the images and the actual site. In order to minimize this discrepancy, a simulation function that integrates AR (augmented reality) objects to the actual site image is necessary. By simulating the current activity progress image to six months into the future and its completed form one year into the future through integration with AR objects, the discrepancy between the 4D object and the actual site can be minimized for the field practitioner.

- Decision-making support system: There is a need to include decision-making functionality that provides activities optimized to the project through integration of analyses of the conventional 4D simulation functionality and risk information, minimization of schedule and workspace overlaps, and optimization functionality including cost optimization. Such functionality minimizes the overlapping schedule in the work zone without changing the total construction time to compare schedules with possible constructability improvement for selective application. Thus, the functionality can be utilized as a useful construction management tool.

\section{Schedule management characteristics for construction project with linear work section}

For construction project with linear work section such as roads and railways, there are many activities that are conducted simultaneously and modifications of manpower and equipment for the construction are necessary depending on the surrounding environment due to external operations. Thus, an activity management solution for 
linear projects is needed. However, although the conventional network method and bar chart are efficient methods of activity management for repetitive activities and limited workspaces, these methods are not appropriate for the management of linear projects, such as roads and railways, with construction sites that span tens of kilometers and include a lot of earthwork. Fig. 1 shows the 3D modeling of the earthwork operation for specific intervals (generally station number) from the starting point to the end point of a railway roadbed construction, which is carried out horizontally.

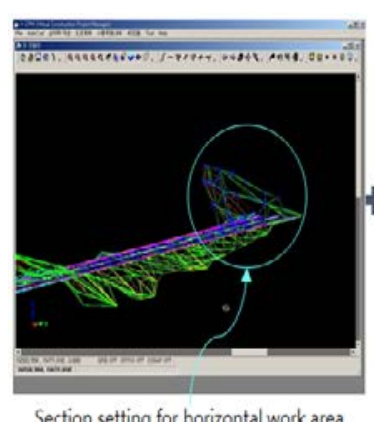

Sertinn settinn for hnrizontal work area

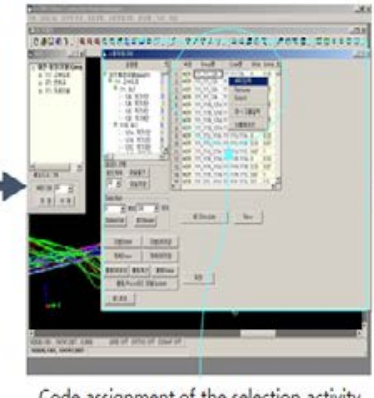

Code assignment of the selection activity

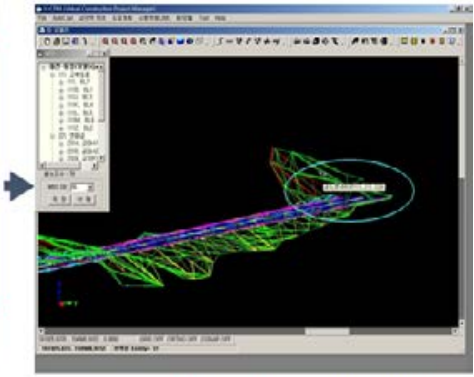

Code representation of the selection activity

Fig. 1. 3D modeling for earthwork activity

The application of 4D simulation is more widely utilized in building construction compared to civil engineering projects, which can be attributed to the greater effectiveness of 4D simulation in terms of establishment and operation since construction work is generally carried out in a relatively vertical manner and is composed of repetitive activities in a small space. On the other hand, in the case of linear facilities such as railways, activities are carried out horizontally and mostly non-repetitive activities are conducted for the construction in a wide workspace, and thus the establishment and operation of 4D simulation is relatively difficult compared to building construction.

In addition, the schedule analysis system used in $4 \mathrm{D}$ CAD system is represented by bar chart or Gantt chart. However, while the conventional network method and bar charts are efficient for the activity management for repetitive activities and limited workspaces, such methods are difficult to implement for the management of linear projects such as railroads and roads with construction sites which span tens of KM. Therefore, it is necessary to integrate a schedule chart representation function specific to the representation of linear activity into the 4D CAD system.

\section{4D modeling for construction project with linear work section}

While a location and schedule data integrated linear project activity management tool (TiLOS, Fig. 2) has been developed and is being used to supplement the existing activity management method for linear facilities, the tool is simply a 2D-based activity management tool that does not provide a visually convenient overview of the detailed location and progress of actual work. Therefore, activity management functionality specialized for linear facilities and a 4D simulation implementation solution is necessary for efficient activity management of linear facilities. For this, an activity management module development methodology, where the location data, schedule data, and their 4D simulation implementation are visualized, as shown in Fig. 3, is proposed in this study. The proposed methodology amplified the advantages and compensated for the disadvantages of the conventional activity management method and 4D simulation, and the methodology is expected to provide an innovative activity management solution for linear projects. 

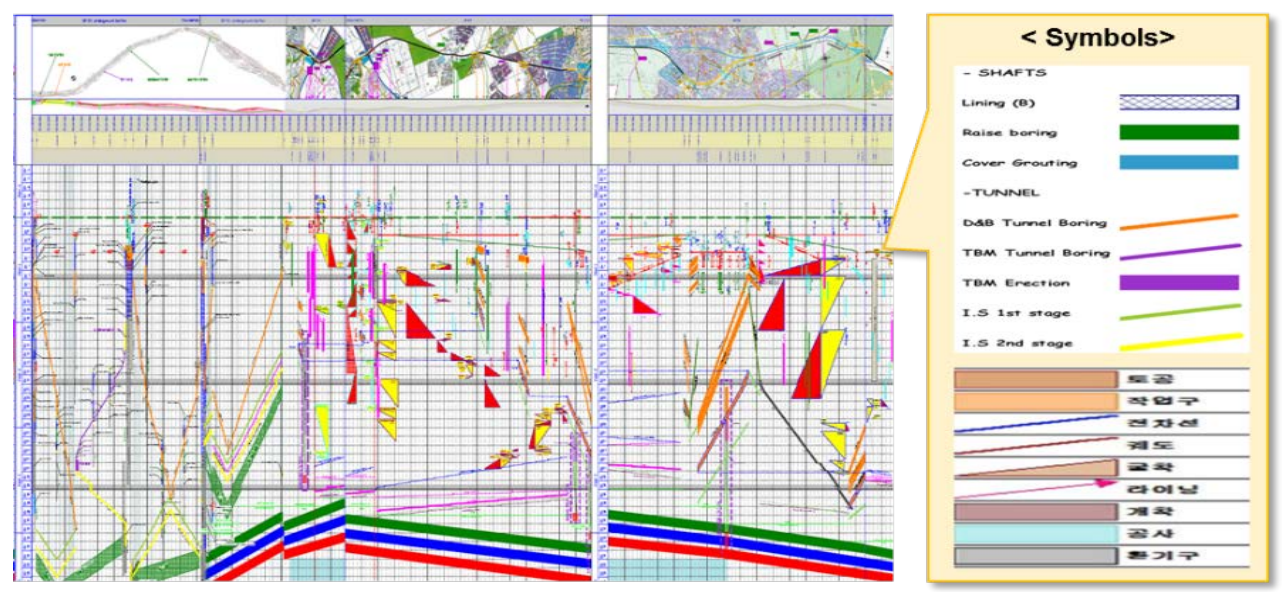

Fig. 2. Example of location-based milestones for railway project (TiLos, 2016)

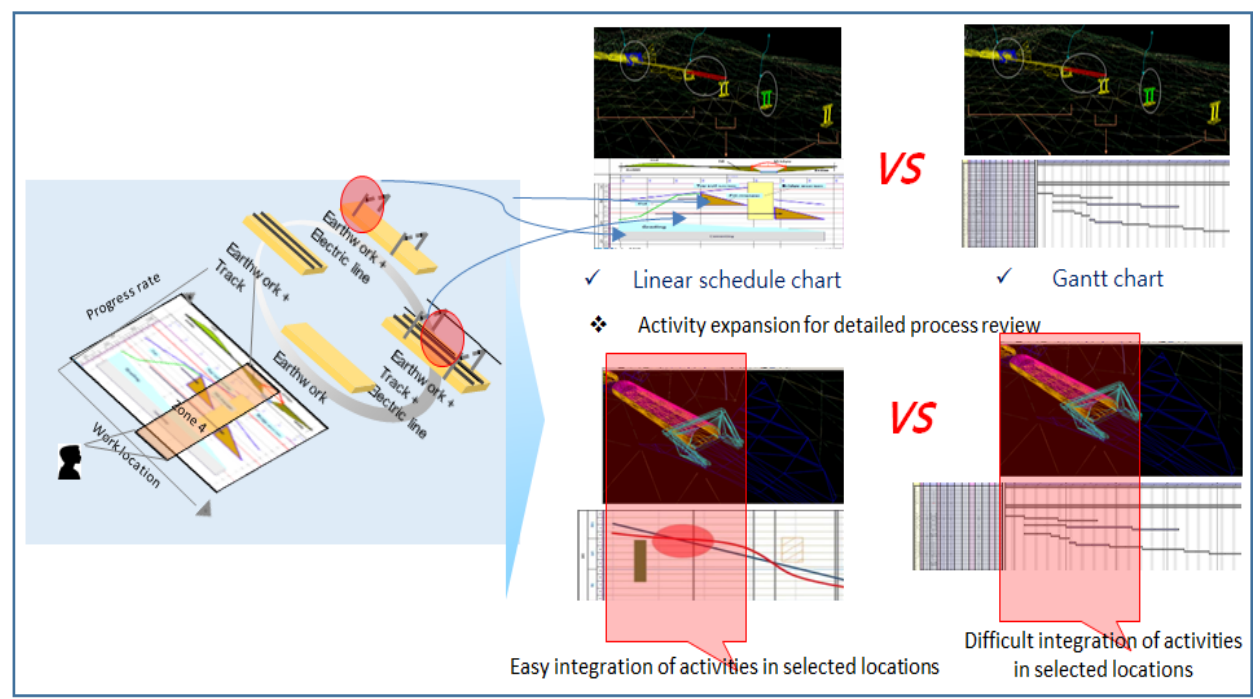

Fig. 3. 4D modeling for linear work sections

The schedule chart represented in the existing 4D CAD system is generally composed of Gantt chart. For the 4D CAD simulation with Gantt chart, showing a simple Gantt chart form of the construction schedule while showing the 3D completed form on the upper portion, as presented in Fig. 4, decreases the practical usability as the distance coordinate for each construction schedule cannot be displayed. To overcome this disadvantage, Fig. 5 shows the linear schedule displayed on the lower portion of the system monitor. The linear schedule simultaneously shows the progress location (X-axis) of each corresponding activity according to the construction schedule (Y-axis) along with the $3 \mathrm{D}$ completed form on the upper portion. 


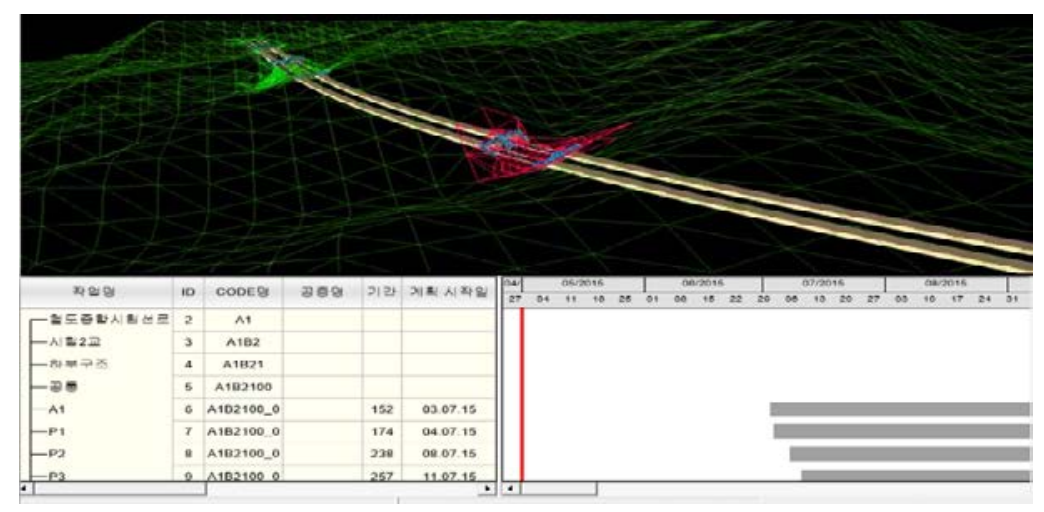

Fig. 4. 4D CAD simulation with Gantt chart

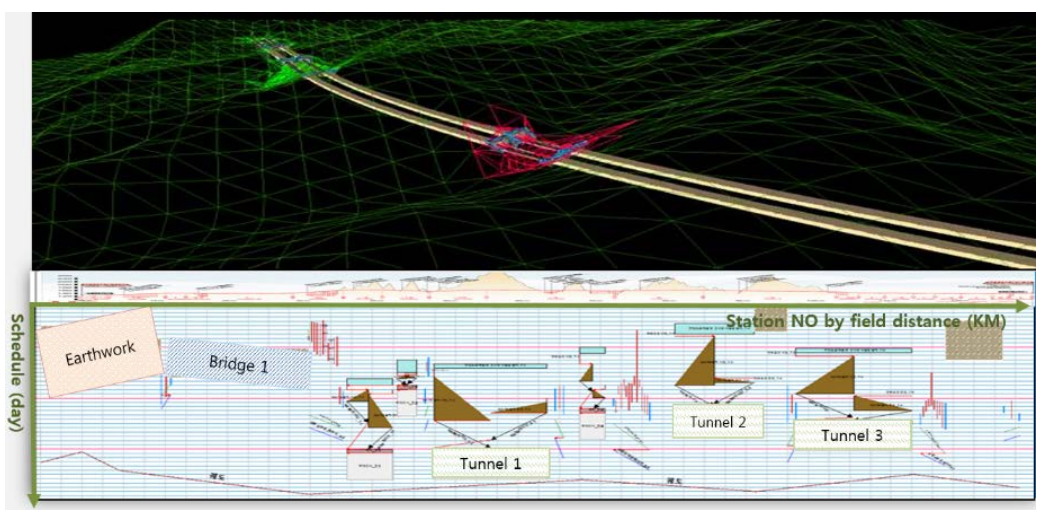

Fig. 5. 4D CAD simulation with linear schedule chart

In the linear schedule-based 4D CAD system, the work location within the project range of the corresponding activity can be simultaneously expressed according to the construction duration carried out in the lower portion linear schedule when 4D objects are simulated on the upper portion. Thus, the location of the corresponding activity in the linear workspace spanning tens of kilometers and the $4 \mathrm{D}$ simulation objects according to the construction time can be simultaneously identified for a linear project, allowing for efficient activity management. Therefore, in order to represent the linear schedule chart, the start and end position information of each activity should be included in addition to the construction schedule information of the project, and the shape applied to the actual project is shown in Fig. 6.

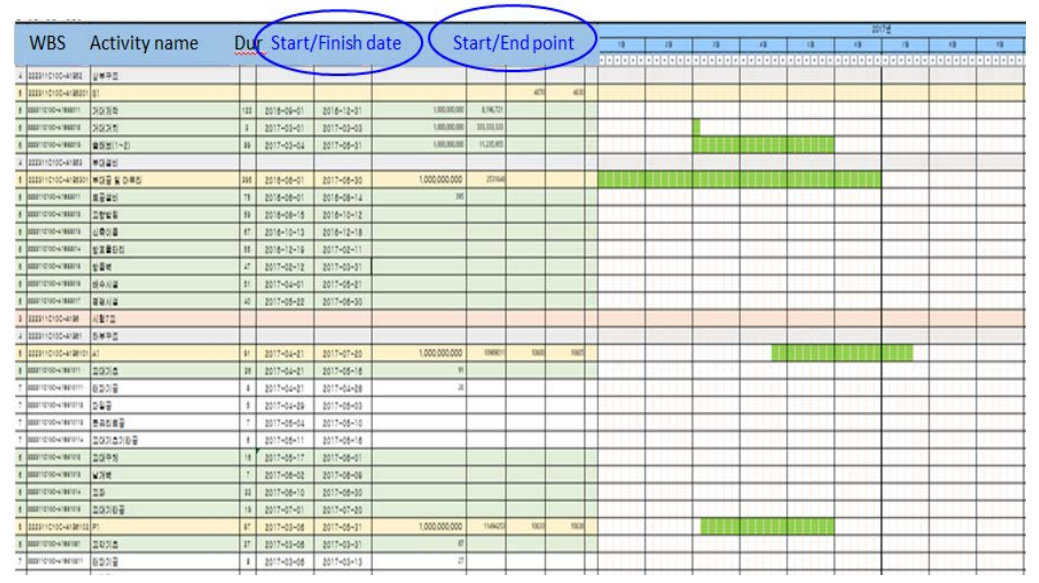

Fig. 6. Input data for schedule activity (including location information) 


\section{Horizontal activity representation in linear schedule chart}

For the implementation of location-based 4D simulation, the activities composed of horizontal tasks need to be generated into a location-based schedule. For the production process of the location-based schedule, as shown in Fig. 7, the start schedule, end schedule, start location, end location, and X-axis reference point were inputted after determining the geometry for the corresponding activity. Based on these input data, the geometry for the corresponding activity is produced on a bar chart. For the location of the geometry generation on the bar chart, the front tip of the corresponding geometry is located at X-axis = start location and $\mathrm{Y}$-axis = start schedule while the rear tip is located at the $\mathrm{X}=$ end location and $\mathrm{Y}=$ end schedule. The $\mathrm{X}$-axis location data were made so that they were automatically converted to reflect the user inputted $\mathrm{X}$-axis reference point.

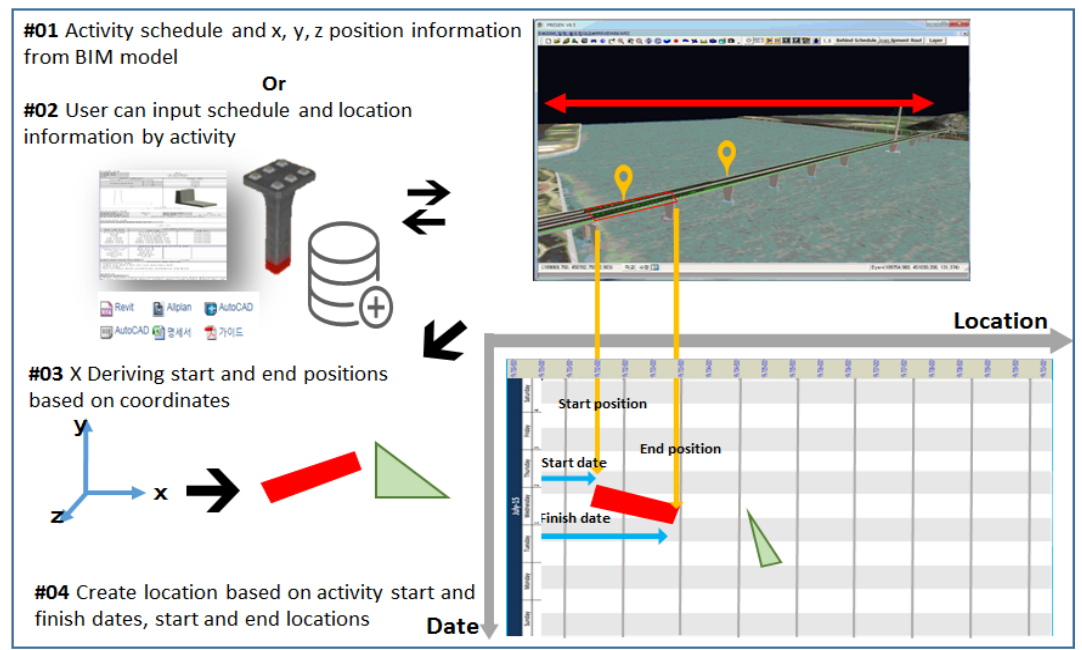

Fig. 7. Location-based linear chart generation for horizontal work process

When the location-based schedule is generated, it can be used to carry out the 4D simulation. The 4D simulation integrated with the location-based schedule is composed of the Y-axis schedule, unlike the conventional Gantt chart schedule. Hence, the "current progress line" moves from top to bottom in the Y-axis direction, as shown in Fig. 7. Since the location-based linear schedule expression method allows the user to conveniently identify the corresponding activity in the schedule and the location of the construction work, it was determined that intuitive activity management is possible using this expression method. In particular, the location-based linear schedule is expected to be more useful when applied to projects with expansive workspaces such as linear projects.

\section{Conclusions}

For efficient activity management of facility construction with activities progressing along the $\mathrm{X}$-axis coordinate in a linear fashion such as roads and railways, the location data of the corresponding activity needs to be shown in addition to the construction time data for each activity. This aspect is differentiated from the activity management of general building construction. In this study, a modeling methodology for linear location-based 4D CAD activity management was proposed. The presented methodology can improve the applicability of the 4D CAD system for civil engineering projects by simultaneously performing the progress simulation according to the schedule of the activity under progress in an expansive construction site and the location data simulation for the activity underway.

\section{Acknowledgements}

The authors would like to thank the Ministry of Land, Infrastructure and Transport of Korea for financially supporting this research under 2018 R\&D program (18RTRP-B122227-04-000000). 


\section{References}

[1] Léon L.olde Scholtenhuis, Timo Hartmann and André G. Dorée. (2016). 4D CAD Based Method for Supporting Coordination of Urban Subsurface Utility Projects. Automation in Construction, 62, pp.66 77.

[2] Christopher Kropp, Christian Koch and MarkusKönig. (2018). Interior construction state recognition with 4D BIM registered image sequences, Automation in Construction, 86, pp.11 32.

[3] Kang, L. S., Moon, H. S., Park, S. Y., Kim, C. H. (2013). "Improved 4D CAD System for Visualizing Construction Work by Using WBS as Interface Code. "KSCE Journal of Civil Engineering, 14(6), pp.803 814.

[4] Moon, H. S, Kim, H. S, Kamat, V. R. and Kang L. S. (2015). "BIM-Based Construction Scheduling Method Using Optimization Theory for Reducing Activity Overlaps. "Journal of Computing in Civil Engineering, 29(3), ASCE

[5] Chin, S. Y., Yoon, S. W., Choi, C. H., and Cho, C. Y. (2008). "RFID+4D CAD for Progress Management of Structural Steel Works in HighRise Buildings. "Journal of Computing in Civil Engineering, 22(2), pp.74 89.

[6] Ashwin, Mahalingam., Rahul, Kashyap., and Charudatta, Mahajan. (2010). "An evaluation of the applicability of 4D CAD on construction projects. "Automation in Construction, 19, pp.148 159.

[7] Timo Hartmann, Ju Gao., and Martin Fischer. (2008), "Areas of Application for 3D and 4D Models on Construction Projects." Journal of Construction Engineering \& Management, 134(10), pp.776 785.

[8] Russell Alan., Sheryl, Staub-French., Ngoc, Tran., and William, Wong. (2009). "Visualizing high-rise building construction strategies using linear scheduling and 4D CAD. "Automation in Construction, 18(2), pp.219 236.

[9] Tilos, https://www.tilos.org(accessed Dec. 2016) 